\title{
Efeito da concentração do amido de milho na liberação de paracetamol de comprimidos
}

\author{
Ana Dóris de Castro', Joice Alessandra Vicente', Samanta Cardozo Mourão², José Hamilton \\ Ferreira Bueno', Raul Cesar Evangelista ${ }^{1}$, Maria Palmira Daflon Gremião ${ }^{1 *}$ \\ ${ }^{1}$ Departamento de Fármacos e Medicamentos, Faculdade de Ciências Farmacêuticas, UNESP, ${ }^{2}$ Núcleo de \\ Investigação Químico Farmacêutica, Curso de Farmácia, Univali, Santa Catarina
}

*Correspondência:

M. P. D. Gremião

Departamento de Fármacos e

Medicamentos

FCF - UNESP

Rodovia Araraquara-Jaú, km 01;

14801-902 - Araraquara - SP

Email: pgremiao@fcfar.unesp.br
Este trabalho avaliou a influência da concentração de amido de milho nas características físicas e na liberação in vitro de paracetamol a partir de comprimidos. Os granulados foram analisados quanto à granulometria e densidades aparentes bruta e compactada e os comprimidos quanto ao peso médio, espessura, dureza, friabilidade, tempo de desintegração. Os comprimidos foram preparados a partir de granulados obtidos por granulação a úmido, utilizando cozimento de amido a 10\% como agente granulante, segundo três formulações. Embora os comprimidos obtidos tenham apresentado características dentro dos limites farmacopéicos, os resultados indicam que variações da concentração de amido provocam diferenças nos diversos parâmetros físicos estudados. Concentração mais alta de amido em pó dá origem, provavelmente, à interação entre os componentes da fórmula, interferindo na liberação in vitro do fármaco. Isto demonstra a importância de se otimizar a concentração dos adjuvantes numa formulação de comprimidos, pois, embora uma pequena variação nesta concentração não exerça efeito significativo no tempo de desintegração, a quantidade de fármaco liberado pode ser substancialmente alterada.
Unitermos:

- Comprimidos

- Amido de milho

- Paracetamol

- Liberação in vitro

\section{INTRODUÇÃO}

Dentre as formas farmacêuticas sólidas o comprimido é uma das mais utilizadas. Quando se dispõe de uma fórmula adequadamente estruturada e de uma técnica de preparo convenientemente otimizada, pode-se considerar que as formas farmacêuticas obtidas por compactação constitu- em preparações farmacêuticas de fácil obtenção. Entretanto, antes de atingir estes estágios, os comprimidos constituem a forma farmacêutica que impõe as maiores dificuldades, as quais estão intimamente relacionadas com a sua formulação e técnica de obtenção (Bueno et al., 1998).

Assim, para se obter comprimidos, ou seja, compactos coesos, é necessário criar condições para que o siste- 
ma particulado sólido tenha predisposição para aglutinabilidade. Paralelamente, é necessário criar condições para que os comprimidos resultantes apresentem homogeneidade de peso, indispensável para garantia de uniformidade de dose. É importante, ainda, o ajuste de parâmetros de formulação e de metodologia com vistas à obtenção de compactos suficientemente resistentes aos choques e atritos desenvolvidos durante o processo produtivo, o transporte a granel e o manuseio pelo usuário, assegurando, assim, a integridade da dose no momento do uso. Finalmente, é necessário ajustar parâmetros de formulação e metodologia para possibilitar a obtenção de comprimidos que apresentem adequada capacidade de liberação do fármaco veiculado, garantindo, desta maneira, a biodisponibilidade e, conseqüentemente, a eficácia terapêutica (Bueno et al., 1998).

Como se observa, muitos são os fatores a serem considerados no desenvolvimento de formulação de comprimidos, sendo comum o aparecimento de dificuldades relativamente acentuadas na concretização desta tarefa. Entretanto, muitos dos aspectos abordados podem ser solucionados de forma relativamente fácil, através do equacionamento dos fatores físicos envolvidos no processo de compressão (Bueno et al., 1998).

Neste trabalho, estudou-se o efeito da concentração de amido de milho na liberação do fármaco paracetamol contido em comprimidos. Para tanto, foram analisados parâmetros como granulometria e densidades aparentes bruta e compactada nos granulados e variação de peso, espessura, dureza, friabilidade, tempo de desintegração e perfil de liberação do fármaco nos comprimidos.

\section{MATERIAL E MÉTODOS}

Neste trabalho foi avaliado o efeito da concentração de amido de milho no processo de liberação do paracetamol, escolhido como fármaco modelo devido à sua reduzida propriedade de compressibilidade e grande possibilidade de desenvolver capping (Fachaux et al., 1995; Garekani et al., 2000; Krycer, Pope, Hersey, 1982; Lennartz, Mielck, 1998).

Foram obtidos comprimidos através do método da granulação a úmido, empregando-se como agente aglutinante/granulante o amido de milho, na forma de cozimento. Primeiramente, foram obtidas as misturas com os três primeiros componentes descritos na Tabela I, através do método da diluição geométrica, com o uso de almofariz e pistilo de porcelana. Em seguida, cada mistura homogênea foi umedecida com quantidade suficiente de cozimento de amido a $10 \%$ para obter grânulos através da passagem da massa úmida pelas malhas do tamis 14 . Os granulados obtidos foram secos em estufa com circulação forçada de ar (Fabbe ${ }^{\circledR}$, modelo 170) até que apresentassem teor de umidade de $1,5 \%$ e, posteriormente, submetidos à análise granulométrica. Com os grânulos, padronizados quanto ao tamanho em tamis 20 , foram obtidos comprimidos em máquina de excêntrico $\left(F a b b e{ }^{\circledR}\right.$, modelo 158 , tamanho grande). Imediatamente antes da compressão, foi incorporada a fase lubrificante constituída por $1 \%$ de talco e $1 \%$ de estearato de magnésio.

TABELA I - Composição das misturas transformadas em comprimidos

\begin{tabular}{lccc}
\hline & \multicolumn{3}{c}{ Fórmula (g) } \\
& A & B & C \\
\hline Paracetamol & 100 & 100 & 100 \\
Lactose & 400 & 200 & 300 \\
Amido (pó) & - & 200 & 100 \\
Cozimento de amido a 10\% & 58 & 116 & 122 \\
\hline
\end{tabular}

Após a calibração, os granulados foram analisados quanto ao tamanho de partícula através de tamisação, empregando-se um vibrador de tamises (Produtest ${ }^{\circledR}$ ), acionado no ponto 8 da escala arbitrária $(0$ a 10$)$ do equipamento. Os tamises utilizados, com suas respectivas aberturas de malha, são apresentados no Quadro 1 (United States Pharmacopeia, 1995). Neste ensaio, cada lote de fórmula $(500 \mathrm{~g})$ foi analisado no todo, tomando-se amostras de no máximo $100 \mathrm{~g}$ de cada vez e repetindo-se o procedimento por 5 vezes.

QUADRO 1 - Número dos tamises e abertura da malha (Farm. Bras., 1988; USP, 1995).

\begin{tabular}{|cc|}
\hline Número do tamis & Abertura da malha (mm) \\
\hline 20 & 0,840 \\
30 & 0,590 \\
40 & 0,420 \\
60 & 0,250 \\
100 & 0,150 \\
\hline
\end{tabular}

As densidades aparentes dos sistemas sólidos particulados foram determinadas, indiretamente, através das medidas de seus volumes aparentes. Para a determinação da densidade aparente bruta, o material foi colocado em uma proveta de $250 \mathrm{~mL}$ do equipamento Tapped Volumeter (Erweka ${ }^{\circledR}$ SVM 12), determinando-se a massa que ocupou este volume bruto em balança semi-analítica 
Ohaus $^{\circledR}$, modelo PL 400. Em seguida, a proveta foi inserida no equipamento e a cada 1250 batidas da proveta, realizaram-se leituras do volume ocupado pelo material. Este procedimento foi repetido até uma redução de $2 \%$ do volume aparente compactado, considerando-se volume compactado aquele lido na penúltima determinação (Badawy et al., 2000; Becker, Rigassi, Bauer-Brandl, 1997; Bolhuis, Eissens, Zoestbergen, 2001; Hancock et al., 2001; Hedenus, et al., 2000; Schmidt, Rubensdörfer, 1994). As densidades aparentes foram calculadas através da seguinte equação:

$$
\begin{aligned}
& \mathrm{d}=\frac{\mathrm{m}}{\mathrm{V}}(\mathrm{g} / \mathrm{mL}), \\
& \text { onde: } \mathrm{d}=\text { densidade }(\mathrm{g} / \mathrm{mL}) \\
& \mathrm{m}=\text { massa }(\mathrm{g}) \\
& \mathrm{V}=\text { volume }(\mathrm{mL})
\end{aligned}
$$

Os comprimidos foram avaliados através dos seguintes ensaios: variação de peso, espessura, dureza, friabilidade, tempo de desintegração e o perfil de liberação do fármaco. A variação de peso dos comprimidos foi determinada por pesagem de 50 unidades de cada lote em balança analítica (Mettler ${ }^{\circledR}$, modelo H31AR). A espessura dos comprimidos foi medida em micrômetro digital (Mitutoyo $^{\circledR}$, precisão de $0,001 \mathrm{~mm}$ ) em 10 unidades de cada lote e em triplicata. A dureza radial foi medida em 50 comprimidos de cada lote em durômetro Schleuniger Pharmatron $^{\circledR}$, modelo 6D. Para o teste de friabilidade empregou-se friabilômetro tipo Roche, Erweka ${ }^{\circledR}$ (modelo TA20). O teste foi realizado de acordo com o preconizado pela Farmacopéia Americana: determinou-se o peso dos 20 comprimidos isentos de pó, antes e depois do rolamento, exprimindo-se a friabilidade em função da percentagem de pó desprendido (United States Pharmacopeia, 1995; Farmacopéia Brasileira, 1988).

No teste de desintegração, determinou-se o tempo necessário para os comprimidos desintegrarem. Foram analisadas 18 unidades de cada lote, em aparelho Erweka ${ }^{\circledR}$ (modelo ZT 502), de acordo com os critérios estabelecidos pelas Farmacopéias Brasileira (Farmacopéia Brasileira, 1988) e Americana (United States Pharmacopeia, 1995).

O perfil de liberação foi determinado empregandose as condições preconizadas pela Farmacopéia Americana, 23 ed. para o teste de dissolução (United States Pharmacopeia, 1995). O meio de dissolução, volume de meio de dissolução, aparelho e velocidade utilizados foram, respectivamente: solução tampão fosfato $\mathrm{pH} 5,8$, $900 \mathrm{~mL}$, cesta e $50 \mathrm{rpm}$. Em intervalos de $5 \mathrm{~min}$ foram retirados $5 \mathrm{~mL}$ do meio de dissolução, que foram imediata- mente repostos. Estas soluções foram filtradas, adequadamente diluídas em tampão fosfato $\mathrm{pH} \mathrm{5,8}$ e as absorvâncias medidas no comprimento de onda de $241 \mathrm{~nm}$. As concentrações de fármaco dissolvido foram calculadas pela equação da curva padrão $\mathrm{y}=0,0264 \mathrm{x}+0,0603$ $\left(r^{2}=0,9986\right)$.

\section{RESULTADOS E DISCUSSÃO}

A Figura 1 mostra a distribuição granulométrica percentual das fórmulas. Nota-se grande semelhança no perfil de distribuição granulométrica das fórmulas A e B. Pode-se observar que, em relação à fórmula $\mathrm{B}$, embora uma grande percentagem dos granulados tenha atravessado as malhas do tamis 100 (grânulos menores que $150 \mu \mathrm{m}$ ), a distribuição granulométrica nos outros tamises foi praticamente equitativa. Estes resultados podem indicar que o aumento da concentração de amido leva a distribuição granulométrica mais homogênea, isto é, os tamanhos de partícula da fórmula B encontram-se distribuídos mais homogeneamente ao longo das faixas de granulometria estudadas. A análise estatística (ANOVA) dos dados confirma a existência de diferença estatisticamente significativa nas frações granulométricas retidas nos tamises 30 (grânulos menores que $840 \mu \mathrm{m}$ e maiores que $590 \mu \mathrm{m}$ ), 60 (grânulos menores que $420 \mu \mathrm{m}$ e maiores que $250 \mu \mathrm{m}$ ), e no coletor (grânulos menores que $150 \mu \mathrm{m}$ ) nos três granulados. Entre as fórmulas B e C não há diferença estatisticamente significante entre as massas retidas no tamis 60 (grânulos menores que $420 \mu \mathrm{m}$ e maiores que $250 \mu \mathrm{m})$. Em relação à menor faixa granulométrica estudada, isto é, partículas menores que $150 \mu \mathrm{m}$ (massa retida no coletor), a fórmula $\mathrm{C}$ apresentou uma percentagem menor que as outras fórmulas. Por outro lado, os grânulos retidos nos tamises 40 (grânulos menores que $590 \mu \mathrm{m}$ e maiores que $420 \mu \mathrm{m}$ ) e 100 (menores que $250 \mu \mathrm{m}$ e maiores que $150 \mu \mathrm{m}$ ) não apresentaram diferenças estatisticamente significativa $(\mathrm{P}=0,067$ e 0,331 , respectivamente).

$\mathrm{O}$ ensaio para determinar as densidades aparentes bruta e compactada foi aplicado às fórmulas após a secagem e o descarte das partículas que ficaram retidas no coletor de tamises. A Tabela II mostra os dados resultantes deste ensaio. A fórmula $\mathrm{B}$ apresenta valores de densidades aparentes, bruta e compactada, menores que as fórmulas A e C. Este resultado está em consonância com os dados de distribuição granulométrica percentual: grânulos maiores apresentaram valores de densidades aparentes mais elevados que os grânulos de menor tamanho. É conhecida a capacidade da lactose para formar ligações interparticulares fortes, o que leva à formação de comprimidos bastante coesos (Cal et al., 1996; Çelik, Driscoll, 


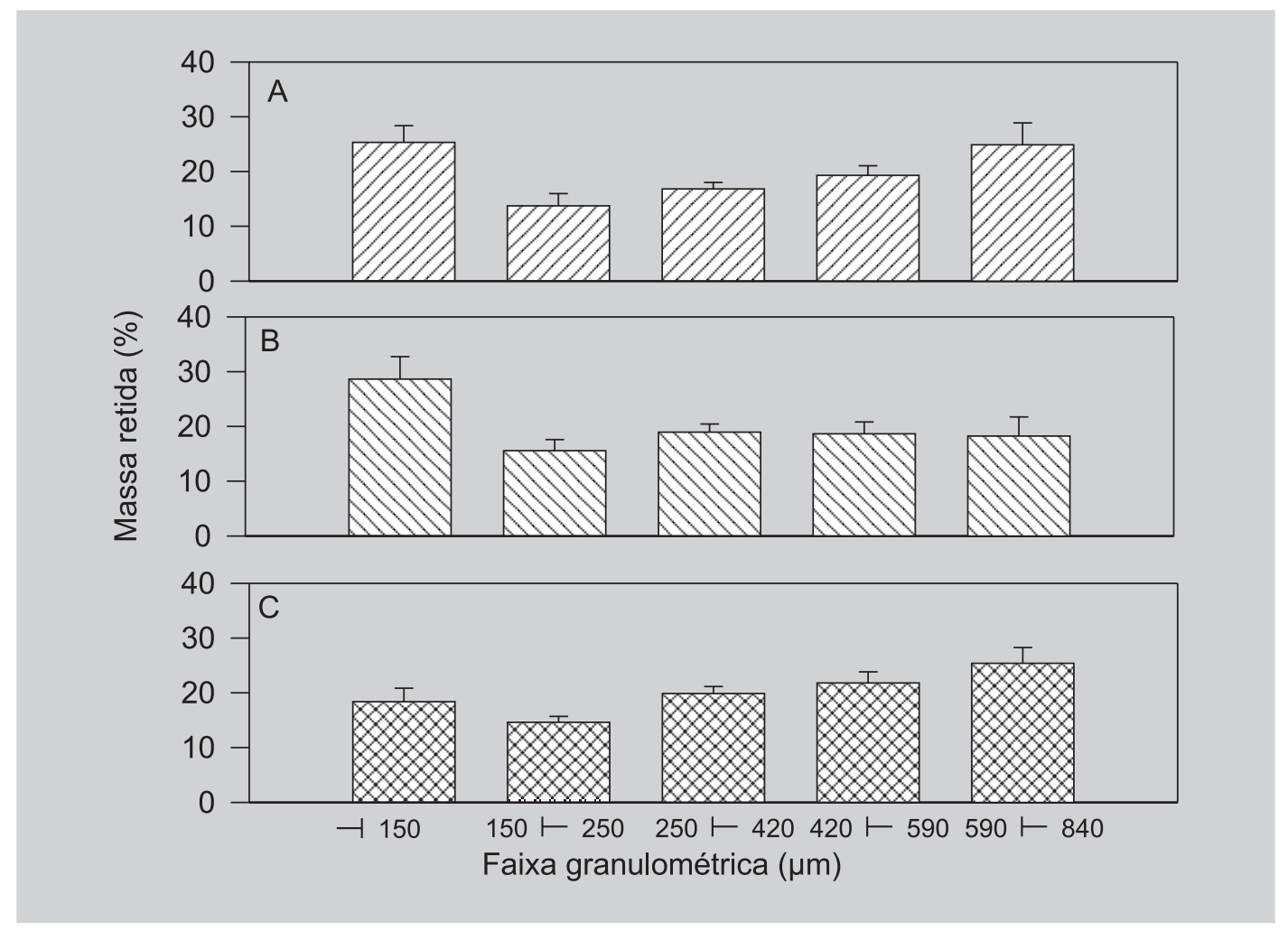

FIGURA 1 - Distribuição granulométrica percentual das fórmulas. Fórmula A: paracetamol $100 \mathrm{~g}$, lactose $400 \mathrm{~g}$, cozimento de amido 58 g; Fórmula B: paracetamol 100 g, lactose 200 g, amido 200 g, cozimento de amido 116 g; Fórmula C: paracetamol $100 \mathrm{~g}$, lactose $300 \mathrm{~g}$, amido $100 \mathrm{~g}$, cozimento de amido $122 \mathrm{~g}(\mathrm{n}=5)$.

TABELA II - Influência da concentração de amido nas densidades aparentes das fórmulas: valores médios e desviopadrão.

\begin{tabular}{lcccc}
\hline & & \multicolumn{2}{c}{ Densidade $(\mathbf{g} / \mathbf{m L})$} & \multicolumn{2}{c}{ Compactada } \\
& média & bruta & média & desvio-padrão \\
\hline Fórmula A & 0,491 & 0,0092 & 0,507 & 0,0105 \\
Fórmula B & 0,446 & 0,0159 & 0,465 & 0,0100 \\
Fórmula C & 0,492 & 0,0106 & 0,515 & 0,0134 \\
\hline
\end{tabular}

Fórmula A: paracetamol 100 g, lactose 400 g, cozimento de amido 58 g; Fórmula B: paracetamol 100 g, lactose 200 g, amido $200 \mathrm{~g}$, cozimento de amido $116 \mathrm{~g}$; Fórmula C: paracetamol $100 \mathrm{~g}$, lactose $300 \mathrm{~g}$, amido $100 \mathrm{~g}$, cozimento de amido $122 \mathrm{~g} ;(\mathrm{n}=3)$.

1993; Sebhatu, Ahlneck, Alderborn, 1997; Vromans, et al., 1986; Zuurman, Bolhuis, Vromans, 1995). Nossa experiência tem mostrado que a lactose tem capacidade de formar grânulos de maior tamanho e densidade na presença de pequena quantidade de amido e que o aumento da concentração de amido leva à obtenção de grânulos menores e menos densos (Castro, 2002). A menor densidade aparente do amido em relação à densidade da lactose pode efetivamente contribuir para tal resultado.
A Tabela III e a Figura 2 mostram a influência da concentração de amido nas características físicas dos comprimidos obtidos, isto é, variação de peso, espessura, dureza, friabilidade e tempo de desintegração.

A câmara de compressão da máquina de comprimir foi ajustada para fornecer comprimidos de pesos aproximados para todas as fórmulas: $0,5158 \mathrm{~g}$ para a fórmula $\mathrm{A}$; $0,5218 \mathrm{~g}$ para a fórmula $\mathrm{B}$ e $0,5224 \mathrm{~g}$ para a fórmula $\mathrm{C}$ (Tabela III). A Farmacopéia Brasileira (1988) permite va- 
riação de 5\% para comprimidos cujo peso teórico é maior que $250 \mathrm{mg}$. Neste estudo, as formulações estudadas apresentaram comprimidos com pesos semelhantes, sob o ponto de vista estatístico, para todas as fórmulas (KruskalWallis One Way Analysis of Variance on Ranks: $\mathrm{P}=$ 0,3272 ). A baixa variação de peso apresentada pelos comprimidos indica que os granulados que os originaram tinham como característica um escoamento adequado e uniforme, pois foram capazes de preencher a câmara de compressão de modo uniforme e constante durante o processo de compressão.

A Tabela III mostra que os comprimidos da fórmula A são mais espessos que os das fórmulas B e C. A análise estatística, Student-Newman-Keuls, destes dados confirma a diferença estatística. A análise dos comprimidos (observação visual) denota superfície lisa e uniforme e espessura condizente com o diâmetro. Como a espessura foi medida, no mínimo, após 24 h da obtenção dos comprimidos, pode-se inferir que os compactos já estavam consolidados e que se houvesse alguma tendência à recuperação elástica esta já teria se manifestado. A maior espessura dos comprimidos da fórmula A parece estar relacionada com o fato de seu peso médio ser maior; entretanto, não se pôde correlacionar este resultado com a densidade aparente das fórmulas, uma vez que os grânulos da fórmula A apresentaram densidades intermediárias. Os valores apresentados para cada fórmula são a média da medição de 10 comprimidos, sendo que cada comprimido foi medido em triplicata em regiões diferentes.

$\mathrm{Na}$ Tabela III pode-se ver os resultados de dureza dos comprimidos. A maior parte dos comprimidos apresentou resistência ao esmagamento (dureza) bastante adequada. Na maior parte dos casos, os valores encontrados foram maiores que $40 \mathrm{~N}$. A inclusão de amido em pó na formulação interfere na dureza dos comprimidos. Pode-se verificar que as fórmulas que contêm amido adicional (B e C) apresentaram valores médios praticamente iguais. A aplicação do teste $t$ confirma esta semelhança entre os valores $(\mathrm{P}=0,801)$. Verifica-se que a fórmula $\mathrm{A}$, que não continha amido em pó, apresentou os menores valores de dureza. A lactose apresenta propriedades aglutinantes e a granulação da fórmula A (sem amido em pó) requereu menor quantidade de agente granulante/aglutinante. Considerando-se a maior capacidade aglutinante da lactose em pó em relação à do amido em pó, esperava-se uma maior dureza nos comprimidos da fórmula $\mathrm{A}$, fato não observado, provavelmente, em função da menor quantidade de agente granulante/aglutinante empregado (Prista, Alves, Morgado, 1995; Sebhatu, Ahlneck, Alderborn, 1997). O ensaio de densidade aparente aplicado às fórmulas resultou em dados conflitantes com os valores de dureza. Podese dizer que os resultados de densidade aparente poderiam levar à expectativa de que os comprimidos com os menores valores de dureza seriam os da fórmula B. Entretanto, deve-se considerar que a densidade aparente de um sistema particulado não é o único fator que influencia as características físicas de um compacto obtido a partir daquele sistema. A interação entre os componentes da fórmula pode ser fator determinante nas características físicas dos compactos.

Os valores de friabilidade (Tabela III), descritos como percentagem de perda de massa após rolamento em equipamento específico, são adequados e extremamente baixos. Todos os lotes foram aprovados no teste preconizado pela Farmacopéia Brasileira (1988), que permite perda máxima de 1,5\%. A Farmacopéia Americana (USP, 1995) é mais exigente e permite perda máxima de $1 \%$; mesmo assim, todas as fórmulas seriam aprovadas de acordo com os critérios adotados pela Farmacopéia Americana.

A resistência às solicitações de ordem mecânica às quais os comprimidos ficam sujeitos após a compressão

TABELA III - Peso médio, espessura, dureza e friabilidade dos comprimidos

\begin{tabular}{lcccc}
\hline & peso médio $(\mathbf{g})$ & espessura $(\mathbf{m m})$ & dureza $(\mathbf{N})$ & friabilidade $(\%)$ \\
\hline Fórmula A & $\overline{\mathrm{x}}: 0,5160$ & $\overline{\mathrm{x}}: 3,6905$ & $\overline{\mathrm{x}}: 39,2$ & $\overline{\mathrm{x}}: 0,12$ \\
& $\sigma: 0,01494$ & $\sigma: 0,06080$ & $\sigma: 10,48$ & $\overline{\mathrm{x}}: 0,32$ \\
Fórmula B & $\overline{\mathrm{x}}: 0,5089$ & $\overline{\mathrm{x}}: 3,6554$ & $\overline{\mathrm{x}}: 45,0$ & \\
& $\sigma: 0,00279$ & $\sigma: 0,88915$ & $\sigma: 2,42$ & $\overline{\mathrm{x}}: 0,97$ \\
Fórmula C & $\overline{\mathrm{x}}: 0,5097$ & $\overline{\mathrm{x}}: 3,6523$ & $\overline{\mathrm{x}}: 45,2$ & \\
& $\sigma: 0,00451$ & $\sigma: 0,59940$ & $\sigma: 2,18$ & \\
\hline
\end{tabular}

Fórmula A: paracetamol $100 \mathrm{~g}$, lactose $400 \mathrm{~g}$, cozimento de amido $58 \mathrm{~g}$; Fórmula B: paracetamol $100 \mathrm{~g}$, lactose $200 \mathrm{~g}$, amido $200 \mathrm{~g}$, cozimento de amido $116 \mathrm{~g}$; Fórmula C: paracetamol $100 \mathrm{~g}$, lactose $300 \mathrm{~g}$, amido $100 \mathrm{~g}$, cozimento de amido $122 \mathrm{~g}$. 
deve ser avaliada considerando-se a dureza e a friabilidade. Assim, todas as fórmulas desenvolvidas neste estudo apresentaram resistência mecânica adequada.

A Figura 2 apresenta os tempos de desintegração de cada formulação estudada. Neste teste, o objetivo foi determinar o tempo exato em que cada comprimido desintegrou, diferentemente do teste farmacopéico, que preconiza um tempo limite de desintegração. Todos os comprimidos desintegraram em tempos extremamente curtos (menos que $2 \mathrm{~min}$ ). Entretanto, a influência do amido como desintegrante tornou-se evidenciada, uma vez que a fórmula que continha a maior concentração de amido, fórmula B, se destacou por apresentar tempo de desintegração menor. A aplicação de análise de variância (StudentNewman-Keuls) confirmou que estas diferenças são estatisticamente significativas $(\mathrm{P}<0,001)$. Estes resultados permitem inferir que menores valores de dureza apresentados pela fórmula A não implicam necessariamente menores tempos de desintegração.

A Farmacopéia Americana (USP, 1995) preconiza que $80 \%$ de paracetamol devem ser liberados a partir de matriz de comprimido em 30 min. Baseando-se em tal critério, apenas a fórmula $\mathrm{C}$ seria aprovada no teste de dissolução. Entretanto, quando se pretende avaliar mais detalhadamente o efeito dos adjuvantes na liberação de um fármaco, é comum estudar-se o perfil de liberação, como no presente caso. Deste modo, o perfil das curvas de liberação do paracetamol é muito semelhante para as três fórmulas, embora as velocidades e a extensão de liberação sejam bem diferentes (Figura 3).
Após 20 min, ambas as fórmulas contendo amido em pó (fórmulas $\mathrm{B}$ e $\mathrm{C}$ ) parecem ter atingido um patamar. Entretanto, a extensão da liberação do paracetamol da fórmula contendo maior concentração de amido em pó (Fórmula B) foi bem menor que a observada com a fórmula C. Considerando que os parâmetros físicos dos comprimidos obtidos a partir das fórmulas $\mathrm{B}$ e $\mathrm{C}$ foram semelhantes (Tabela III) e a desintegração não é um fator limitante para a liberação do paracetamol, uma vez que os tempos de desintegração foram curtos, pode-se sugerir que a interação entre os componentes da fórmula interfira na liberação do paracetamol da fórmula B. Concentração maior de amido em pó (fórmula B) dificultou a liberação do paracetamol, sugerindo, neste caso, possível interação entre amido e paracetamol. Deve-se considerar ainda a possibilidade de interação entre amido e lactose, a qual foi comprovada através de técnica termoanalítica por Robusti (2001), como causa mais provável da diminuição da velocidade e extensão de liberação do paracetamol. A fórmula na qual o amido foi utilizado apenas como agente aglutinante/granulante (Fórmula A) apresentou velocidade e extensão de liberação intermediárias àquelas observadas nas outras fórmulas (B e C). Neste caso, o efeito da dissolução da lactose parece prevalecer, facilitando a liberação do paracetamol. Entretanto, quando o amido está em maior concentração (Fórmula B), a interação mais intensa entre lactose e amido pode contribuir para a redução da taxa de dissolução da lactose ou, ainda, do efeito desintegrante do amido.

Este estudo mostra a importância de se otimizar a

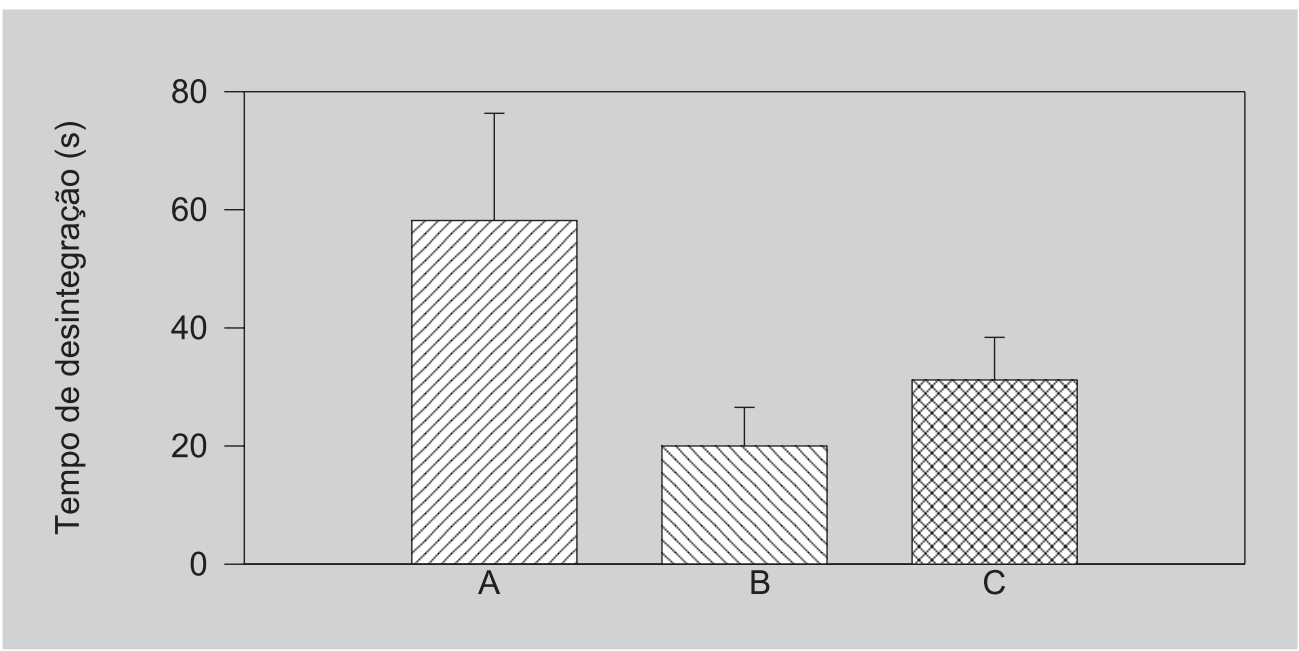

FIGURA 2 - Influência da concentração de amido de milho no tempo de desintegração de comprimidos de paracetamol. Fórmula A: paracetamol $100 \mathrm{~g}$, lactose $400 \mathrm{~g}$, cozimento de amido $58 \mathrm{~g}$; Fórmula B: paracetamol $100 \mathrm{~g}$, lactose $200 \mathrm{~g}$, amido $200 \mathrm{~g}$, cozimento de amido $116 \mathrm{~g}$; Fórmula C: paracetamol $100 \mathrm{~g}$, lactose $300 \mathrm{~g}$, amido $100 \mathrm{~g}$, cozimento de amido $122 \mathrm{~g}$. 


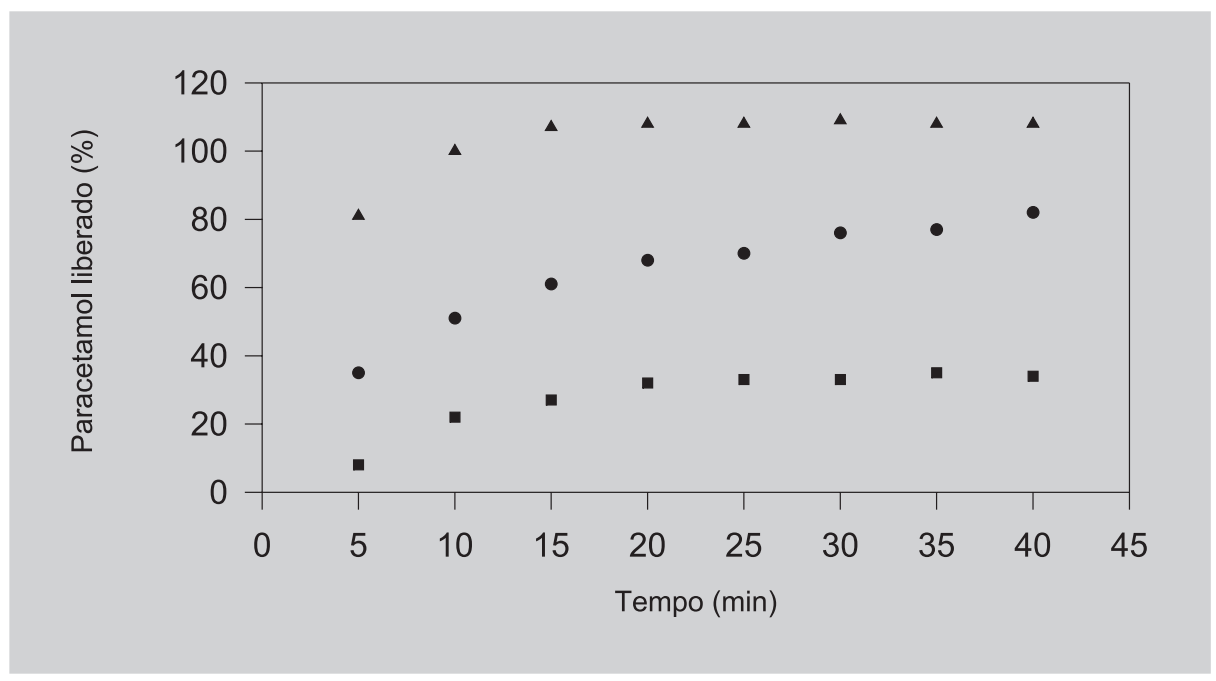

FIGURA 3 - Influência da concentração de amido no perfil de liberação do paracetamol. O: Fórmula A (paracetamol $100 \mathrm{~g}$, lactose $400 \mathrm{~g}$, cozimento de amido $58 \mathrm{~g}$ ) $\mathbf{\square}$ : Fórmula B (paracetamol $100 \mathrm{~g}$, lactose $200 \mathrm{~g}$, amido $200 \mathrm{~g}$, cozimento de amido $116 \mathrm{~g}$ ); $\boldsymbol{\Delta}$ : Fórmula C (paracetamol $100 \mathrm{~g}$, lactose $300 \mathrm{~g}$, amido $100 \mathrm{~g}$, cozimento de amido $122 \mathrm{~g}$ ); $\mathrm{n}=6$.

concentração dos componentes da formulação. Pequena variação nesta concentração pode não exercer efeito significativo no tempo de desintegração. Entretanto, a velocidade e extensão de fármaco liberado pode ser substancialmente alterada em conseqüência desta modificação. Segundo levantamento realizado em 1999 pelo Food and Drug Administration, problemas relacionados à liberação do fármaco estiveram entre as principais causas de recolhimento de medicamentos (FDA-CDER).

\section{CONCLUSÃO}

Todas as fórmulas desenvolvidas neste estudo apresentaram características físicas adequadas em relação aos parâmetros farmacopéicos. Os comprimidos apresentaram baixa variação de peso, espessura uniforme, valores de dureza e friabilidade satisfatórios e tempos de desintegração curtos. Dependendo da concentração, o amido em mistura com lactose pode alterar as características de liberação do paracetamol em comprimidos.

\section{ABSTRACT \\ Effect of maize starch concentration on in vitro acetaminophen release from tablets}

This paper describes the influence of maize starch concentration on the physical characteristics and on in vitro release of acetaminophen from compressed tablets. The granulates were analyzed in relation to size distribution and bulk and compacted densities, and the tablets in relation to mean weight, thickness, hardness, friability and disintegration time. The tablets were prepared from granulates made by wet granulation with $10 \%$ starch paste in three formulations. Although the tablets obtained have presented characteristics in accordance with pharmacopeial limits, the results indicate that variations on starch concentration cause differences on the several physical parameters studied. Higher starch concentration probably originates an interaction among the ingredients of the formulations, interfering on the in vitro drug release. This fact demonstrates the importance in optimizing the concentration of the adjutants in a tablet formulation, because, although a short variation in this concentration has no significant effect on disintegration time, the amount of released drug can be substantially modified.

UNITERMS: Tablets. Maize starch. Acetaminophen. In vitro release.

\section{SUPORTE FINANCEIRO}

PADC-FCF-Unesp.

\section{REFERÊNCIAS BIBLIOGRÁFICAS}

BADAWY, S. I. F.; MENNING, M. M.; GORKO, M. A.; GILBERT, D. L. Effect of process parameters on compressibility of granulation manufactured in a highshear mixer. Int. J. Pharm., Amsterdam, v.198, n. 1, p. 51$61,2000$. 
BECKER, D.; RIGASSI, T.; BAUER-BRANDL, A. Effectiveness of Binder in Wet Granulation: A Comparison Using Model Formulations of Different Tabletability. Drug Dev. Ind. Pharm., Monticello, v. 23, n. 8, p. 791-808, 1997.

BOLHUIS, G. K.; EISSENS, A. C.; ZOESTBERGEN, E. DC Calcium lactate, a new filler-binder for direct compaction of tablets. Int. J. Pharm., Amsterdam, v. 221, n. 1-2, p.77-86, 2001.

BUENO, J. H. F.; CASTRO, A. D.; EVANGELISTA, R. C.; GREMIÃO, M. P. D. Alguns fatores físicos envolvidos na compressão e suas aplicações na formulação de comprimidos. Rev. Ciênc. Farm., São Paulo, v. 19, n. 2, p.183-196, 1998.

CAL, S.; RODRÍGUEZ-PUENTE, B.; SOUTO, C.; CONCHEIRO, A.; GÓMEZ-AMOZA, J. L.; MARTÍNEZ-PACHECO, R. Comparison of a spraydried $a$-lactose monohydrate with a fully hydrated rollerdried $b$-lactose. Int. J. Pharm., Amsterdam, v.136, p. 1321, 1996.

CASTRO, A. D. Farelo de mandioca: Avaliação do potencial de utilização como desintegrante em comprimidos. 2002. 114. [Tese de Doutorado - Faculdade de Ciências Farmacêuticas - Universidade Estadual Paulista "Julio de Mesquita Filho"].

ÇELIK, M.; DRISCOLL, C. E.; An overview of the effects of some physico-chemical and mechanical characteristics of particulates on the compaction and post-compaction properties of compacts. Drug Dev. Ind. Pharm., Monticello, v. 19, p. 2119-2141, 1993.

FACHAUX, J. M.; GUYOT-HERMANN, A.-M.; GUYOT, J.-C.; CONFLANT, P.; DRACHE, M.; VEESLER, S.; BOISTELLE, R. Pure paracetamol for direct compression. Part I. Development of sintered-like crystals of Paracetamol. Powder Technol., Amsterdam, v. 82, n. 2, p. 123-128, 1995.

FARMACOPÉIA BRASILEIRA. 4 ed. São Paulo: Atheneu Editora São Paulo, pt. 1, p.v.1.1., v.1.1.-2.,v.1.3.-1.3.2., v.1.4.1., v.2.1.1., v.2.11-2, 1988.

FDA-US - Food and Drug Administration - Center for Drug Evaluation and Research. Disponível em: http:// www.fda.gov/cder/handbook/index.htm. Acesso em 18 out. 2002 .
GAREKANI, H. A.; FORD, J. L.; RUBINSTEIN, M. H.; RAJABI-SIAHBOOMI, A. R. Highly compressible paracetamol - II. Compression properties. Int. J. Pharm., Amsterdam, v. 208, n. 1-2, p. 101-110, 2000.

HANCOCK, B.; CARLSON, G. T.; LADIPO, D. D.; LANGDON, B. A.; MULLARNEY, M. P. The powder flow and compact mechanical properties of two recently developed matrix-forming polymers. J. Pharm. Pharmacol., London, v. 53, p. 1193-1199, 2001.

HEDENUS, P.; MATTSSON, M. S.; NIKLASSON, G. A.; CAMBER, O.; EK, R. Characterisation of instantaneous water absorption properties of pharmaceutical excipients. Int. J. Pharm., Amsterdam, v. 202, n. 1-2, p. 141-149, 2000.

KRYCER, I.; POPE, D. G.; HERSEY, J. A. The prediction of paracetamol capping tendencies. J. Pharm. Pharmacol., Wallinghard, v. 34, p. 802-804, 1982.

LENNARTZ, P.; MIELCK, J. B. Minitabletting: improving the compactibility of paracetamol powder mixtures. Int. J. Pharm., Amsterdam, v. 173, n. 1-2, p. 75-85, 2000.

PRISTA, L N., ALVES, A. C., MORGADO, R. M. R., Técnica Farmacêutica e Farmácia Galênica, 4 ed., Lisboa: Fundação Calouste Gulbenkian, 1992. p.607608, 627-31, 659-784.

ROBUSTI, L. M. G. Verificação de interação excipienteexcipiente em formulação de comprimidos, através de análise térmica-DSC. 2001.2001. 131. [Dissertação de Mestrado - Faculdade de Ciências Farmacêuticas Universidade Estadual Paulista "Julio de Mesquita Filho"].

SEBHATU, T.; AHLNECK, C.; ALDERBORN, G. The effect of moisture content on the compression and bondformation properties of amorphous lactose particles. Int. J. Pharm., Amsterdam, v. 147, p. 101-114, 1997.

SCHMIDT, P. C.; RUBENSDÖRFER. Evaluation of Ludipress as "Multipurpose excipient" for direct compression. Part 1: Powder characteristics and tableting properties. Drug Dev. Ind. Pharm., Monticello, v. 20, n. 18, p. 2899-2925, 1994.

UNITED STATES PHARMACOPEIA. 23. ed. rev. Rockville: United States Pharmacopeial, 1995. p.17901793, p.1822-1823. 
VROMANS, H.; BOLHUIS, G. K.; LERK, C. F.; KUSSENDRAGER, K. D. Studies on tableting properties of lactose. VI. Consolidation and compaction of spray dried amorphous lactose. Acta Pharm. Suec., Stockholm, v.23, p. 231-240, 1986.
ZUURMAN, K.; BOLHUIS, G. K.; VROMANS, H. Effect of binder on the relationship between bulk desntiy and compactibility of lactose granulations. Int. J. Pharm., Amsterdam, v. 119, n. 1, p.65-69, 1995.

Recebido para publicação em 12 de fevereiro de 2003. 